\title{
The piRNA pathway is developmentally regulated during spermatogenesis in Drosophila
}

\author{
EMILIE QUÉNERCH'DU, ${ }^{1,2,3}$ AMIT ANAND, ${ }^{1,3}$ and TOSHIE KAI ${ }^{1,4}$ \\ ${ }^{1}$ Temasek Life Sciences Laboratory, 1 Research Link, National University of Singapore, 117604 Singapore, Singapore \\ ${ }^{2}$ Department of Biological Sciences, National University of Singapore, 117543 Singapore, Singapore
}

\begin{abstract}
PIWI-interacting RNAs (piRNAs) are predominantly produced in animal gonads to suppress transposons during germline development. Our understanding about the piRNA biogenesis and function is predominantly from studies of the Drosophila female germline. piRNA pathway function in the male germline, however, remains poorly understood. To study overall and stage-specific features of piRNAs during spermatogenesis, we analyzed small RNAs extracted from entire wild-type testes and stage-specific arrest mutant testes enriched with spermatogonia or primary spermatocytes. We show that most active piRNA clusters in the female germline do not majorly contribute to piRNAs in testes, and abundance patterns of piRNAs mapping to different transposon families also differ between male and female germlines. piRNA production is regulated in a stage-specific manner during spermatogenesis. The piRNAs in spermatogonia-enriched testes are predominantly transposon-mapping piRNAs, and almost half of those exhibit a ping-pong signature. In contrast, the primary spermatocyte-enriched testes have a dramatically high amount of piRNAs targeting repeats like suppressor of stellate and AT-chX. The transposon-mapping piRNAs in the primary spermatocyte stages lacking Argonaute 3 expression also show a ping-pong signature, albeit to a lesser extent. Consistently, argonaute 3 mutant testes also retain ping-pong signature-bearing piRNAs, suggesting that a noncanonical pingpong cycle might act during spermatogenesis. Our study shows stage-specific regulation of piRNA biogenesis during spermatogenesis: An active ping-pong cycle produces abundant transposon-mapping piRNAs in spermatogonia, while in primary spermatocytes, piRNAs act to suppress the repeats and transposons.
\end{abstract}

Keywords: spermatogenesis; Drosophila; piRNA; germline; RNA silencing; PIWI proteins

\section{INTRODUCTION}

Germline cells pass genetic information to the next generation in sexually reproducing organisms. The integrity of the genome of the germline cells is carefully maintained in order to produce healthy progeny. Transposons are a major threat to the integrity of the germline genome: They have the ability to insert themselves at new locations in host genomes and can compromise genome integrity by disrupting the genome sequence (McClintock 1951; Lim et al. 2009). The transposons in animal gonads are silenced by a conserved small RNA-based defense mechanism involving the PIWI family proteins and associated small RNAs that are 23-29 nucleotides (nt) long, called PIWI-interacting RNAs (piRNAs) (Aravin et al. 2006; Girard et al. 2006; Grivna et al. 2006; Lau et al. 2006; Brennecke et al. 2007). In Drosophila gonads, the biogenesis of piRNAs requires three PIWI family pro-

\footnotetext{
${ }^{3}$ These authors contributed equally to this work.

${ }^{4}$ Present address: Graduate School of Frontier Biosciences, Osaka University, Suita, Osaka 565-0871, Japan

Corresponding author: toshie_kai@fbs.osaka-u.ac.jp

Article published online ahead of print. Article and publication date are at http://www.rnajournal.org/cgi/doi/10.1261/rna.055996.116.
}

teins: Piwi, Aubergine (Aub), and Argonaute3 (Ago3) (Cox et al. 2000; Harris and Macdonald 2001; Brennecke et al. 2007). These three proteins together with their associated piRNAs ensure the repression of transposons through transcriptional and post-transcriptional silencing (Brennecke et al. 2007; Lim et al. 2009; Le Thomas et al. 2013).

Production of piRNAs in Drosophila ovarian germline cells consists of a primary pathway and an amplification loop termed ping-pong cycle. The primary pathway is a process where long piRNA precursor transcripts are parsed into shorter fragments, loaded onto PIWI proteins and further processed into mature primary piRNAs (Brennecke et al. 2007; Kawaoka et al. 2011). The ping-pong cycle is a secondary pathway that is initiated by primary piRNAs. In this cycle, Aub, guided by an antisense piRNA, can target a sense transcript from an active transposon and slices it by endonucleolytic cleavage. This cleavage on the sense transcripts occurs precisely $10 \mathrm{nt}$ downstream from uracil $(\mathrm{U})$ at the $5^{\prime}$ end of

C 2016 Quénerch'du et al. This article is distributed exclusively by the RNA Society for the first 12 months after the full-issue publication date (see http:// rnajournal.cshlp.org/site/misc/terms.xhtml). After 12 months, it is available under a Creative Commons License (Attribution-NonCommercial 4.0 International), as described at http://creativecommons.org/licenses/by-nc/4.0/. 
the antisense piRNA, generating a 10-nt overlap between the sense and antisense piRNAs as well as a bias for adenine (A) at the 10th position of the sense piRNA. The sense piRNA intermediate is then loaded onto Ago 3 and further processed for maturation. In turn, Ago3 with mature sense piRNA can slice antisense piRNA precursors, thus producing the $5^{\prime}$ end of the antisense piRNA to be loaded onto Aub, and the cycle continues (Brennecke et al. 2007; Gunawardane et al. 2007).

Two PIWI family proteins, Aub and Ago3, involved in the ping-pong cycle, localize to the nuage in germline cells (Harris and Macdonald 2001; Brennecke et al. 2007; Gunawardane et al. 2007). Nuage (which means "cloud" in French) is a conserved perinuclear structure present at the cytoplasmic face of the nuclear envelope in germline cells (Eddy 1975). Interestingly, in Drosophila, many of the nuage proteins have been involved in the production of piRNAs, such as DEAD-box RNA helicases like Vasa (Liang et al. 1994), PIWI family proteins Aub and Ago3 (Harris and Macdonald 2001; Brennecke et al. 2007; Gunawardane et al. 2007), and many Tudor domain proteins such as Krimper (Krimp) (Lim and Kai 2007; Sato et al. 2015) and Qin/Kumo (Zhang et al. 2011; Anand and Kai 2012). Thus, nuage was proposed as the potential site for a ping-pong amplification loop in the germline.

While the biogenesis and function of piRNAs have been extensively studied using Drosophila female germline cells, they remain poorly understood in the male germline. The Drosophila testis contains 6-9 germline stem cells (GSCs) that are maintained in a microenvironment known as the niche (Hardy et al. 1979; Spradling et al. 2008). GSCs divide asymmetrically to give rise to a GSC and a gonialblast. The gonialblast undergoes four mitotic divisions to form a 16-cell interconnected cyst known as spermatogonia. Spermatogonia then differentiate into primary spermatocytes. At this stage, the spermatocytes switch into a state of growth and gene expression (Fig. 1A; Fuller 1993). In Drosophila ovaries, Aub and Ago3 are expressed in germline cells of all stages except for quiescent oocytes and are proposed to act together for efficient piRNA production via the ping-pong cycle (Brennecke et al. 2007; Li et al. 2009; Malone et al. 2009). In contrast, expression of PIWI family protein Ago3 is not observed during later stages of spermatogenesis (Fig. 1B; Nagao et al. 2010). The unique spatial expression of PIWI family proteins implicates different modes of piRNA biogenesis during spermatogenesis. Previous studies showed that Aub in Drosophila testes associates with two predominant classes of piRNAs: One derives from the Suppressor of Stellate $[\mathrm{Su}(\mathrm{Ste})]$ repeats on the Y chromosome, named Su (Ste) piRNAs, and the other derives from the $A T$-chX region on the $\mathrm{X}$ chromosome, named $A T$-chX piRNAs (Nishida et al. 2007; Nagao et al. 2010). The $S u$ (Ste) piRNAs suppress Ste expression, and perturbation of piRNA pathway results in the formation of Ste protein crystals in primary spermatocytes (Bozzetti et al. 1995; Aravin et al. 2001, 2004; Kotelnikov et al. 2009). AT-chX piRNAs have strong complementarity to vasa (vas) mRNA, and aub mutant testes exhibits increased Vas expression, implicating that vas may be a target of $A T$-chX piRNAs in the male germline (Nishida et al. 2007). Previous studies indicated that $S u(S t e)$ and $A T$ chX piRNAs derive mostly from antisense precursors and that their production through a ping-pong cycle is unlikely (Nagao et al. 2010). In contrast, $>50 \%$ Ago3 bound piRNAs in testes are mapped to transposons (Nagao et al. 
2010). These findings suggest that Aub and Ago3 may be involved in piRNA biogenesis in additional ways apart from ping-pong amplification of transposon-derived piRNAs during Drosophila spermatogenesis (Nishida et al. 2007; Nagao et al. 2010). However, the details of piRNA biogenesis during spermatogenesis remained elusive.

In order to probe piRNA dependent suppression of repetitive elements and transposons in the male germline, we analyzed piRNA pathway components expression and piRNA populations during spermatogenesis. Using mutant testes containing stage-arrested germline cells, we conclusively show that Aub and Ago3 exhibited distinct expression patterns during spermatogenesis. While Aub was expressed broadly from GSCs to primary spermatocytes, Ago3 was detected only in mitotically dividing germline cells (GSCs and spermatogonia), indicating stage-specific modulations of the piRNA pathway. Analysis of stage-specific piRNAs isolated from stage-arrested mutant testes revealed that distinct piRNA populations are expressed at different stages. We observed that piRNAs with the ping-pong signature are more abundant in spermatogonia albeit still detectable in primary spermatocytes where Ago 3 is not expressed. These results suggest that robust piRNA production via the ping-pong cycle takes place in spermatogonia, and to a lesser extent in primary spermatocytes even in the absence of Ago3. Consistently, we also detected piRNAs with a ping-pong signature from ago3 mutant testes, suggesting that a noncanonical ping-pong cycle acts during spermatogenesis. This work illustrates the high versatility of the piRNA pathway and indicates a novel developmental dimension of the piRNA-based mechanisms.

\section{RESULTS}

\section{Aub and Ago3 exhibit distinct expression patterns during spermatogenesis}

In order to characterize the piRNA pathways in the male germline, we first examined the expression pattern of Aub and Ago3 during spermatogenesis by immunostaining. In agreement with a previous report, Aub and Ago3 localized to perinuclear foci known as nuage in the male germline (Fig. 1B; Eddy 1975; Nagao et al. 2010). However, unlike their uniform expression during oogenesis, Aub and Ago3 exhibited a stage-specific expression pattern during spermatogenesis (Fig. 1B; Brennecke et al. 2007). Though Aub was expressed widely from GSCs to primary spermatocytes, Ago3 was detected only in the gonial proliferation center (GPC) where germline cells divide mitotically, i.e., in GSCs and spermatogonia, but not in primary spermatocytes. Expression of Aub alone in primary spermatocytes suggests a distinct mechanism of piRNA generation.

The distinct expression patterns of the PIWI family proteins prompted us to examine stage-specific characteristics of the piRNA pathway during spermatogenesis. In order to obtain testicular samples enriched with male germline cells representing different stages of spermatogenesis, we used developmentally arrested mutant testes: "bag of marbles" (bam) and "benign gonial cell neoplasm" (bgcn), where testes predominantly contain GSCs and spermatogonia, and "cannonball" (can) and "spermatocyte arrest" (sa), where testes are enriched with primary spermatocytes. In bam and bgcn mutant testes, germline cells are unable to differentiate into primary spermatocyte, resulting in testes enriched with spermatogonia (McKearin and Ohlstein 1995; Gonczy et al. 1997). Besides having bright nuclear staining with DAPI, the early germline cells accumulating in bam and $b g c n$ mutants have branched fusomes as revealed by immunostaining with the fusome marker, Hu-li tai shao (Hts) (Supplemental Fig. S1A; Lin et al. 1994). In addition, in the bam and $b g c n$ mutant testes, qRT-PCR failed to detect the expression of the male fertility genes, $k l-5$ and $k l-3$ and ory, which are known to be expressed in the primary spermatocytes (Supplemental Fig. S1B; Meyer et al. 1961). Together, these results suggest that the early germline cells accumulating in bam and bgcn testes most resemble spermatogonia. In contrast, to obtain testes enriched with primary spermatocytes, we used mutants of can and sa, which encode testicular TBP-associated factors (tTAFs) required for the entry into the first meiotic division (Hiller et al. 2004; Chen et al. 2005). The germline cells in can and sa mutant testes cannot progress beyond the primary spermatocyte stage, resulting in an enrichment of spermatocytes expressing a spermatocyte marker, S5 (Supplemental Fig. S1C; Risau et al. 1983). To examine the functionality of the piRNA pathway in these developmentally arrested mutants, we first examined the expression of Ste, which is silenced by the piRNA pathway during spermatogenesis (Bozzetti et al. 1995; Aravin et al. 2001, 2004; Kotelnikov et al. 2009). In wild-type and all the examined developmentally arrested mutant testes, Ste expression could not be detected, while we observed Ste crystals in mutant testes of a robust piRNA pathway component, aub (Supplemental Fig. S2A), indicating that Ste is properly silenced via piRNA pathway in those developmentally arrested testes. In addition, developmentally arrested testes did not show de-repression of transposons, such as 1360 and $m d g 3$, which are severely up-regulated in $a u b$ mutant testes (Supplemental Fig. S2B). These results suggest that the piRNA pathway remains functional to silence transposons and other repetitive elements in these developmentally arrested mutant testes. It has been reported that localization of piRNA pathway components to the perinuclear nuage, the site for piRNA biogenesis via the ping-pong cycle, is often perturbed in the germline cells of piRNA pathway mutants (Findley et al. 2003; Lim and Kai 2007; Li et al. 2009; Patil and Kai 2010; Anand and Kai 2012). In developmentally arrested mutant testes, however, the PIWI family proteins, Aub and Ago3, remained localized to their characteristic perinuclear foci (Supplemental Fig. S3). Both Aub and Ago3 staining exhibited perinuclear foci in undifferentiated germline cells (from apex to distal part in bam and bgcn mutant testes, 
and at the apex of can and sa testes). Like in wild-type, in the arrested primary spermatocytes of can and sa testes, only Aub but not Ago3 was detected at the perinuclear foci. These results suggest that bam, bgcn, can, and sa mutant testes have a functional piRNA pathway.

To investigate the dynamics of the piRNA pathway during spermatogenesis, we used whole bam and bgcn mutant testes enriched with spermatogonia, while we manually removed the apical regions of can and $s a$ mutant testes to obtain samples enriched with primary spermatocytes devoid of undifferentiated germline cells (Fig. 1C). The use of the bam, $b g c n, c a n$, and sa mutant testes allowed us to investigate the stage-specific characteristics of the piRNA populations at the spermatogonia and primary spermatocyte stages. Consistent with the immunostaining, we detected Aub expression in testicular extracts from the wild-type control yellow white $(y w)$, mutants enriched with spermatogonia, bam and $b g c n$, and mutants enriched with primary spermatocytes, can and sa, by Western blot (Fig. 1D). In contrast, Ago3 was detected in $y w, b a m$, and $b g c n$, but not in can and sa testes devoid of apex (Fig. 1D).

\section{Distinct piRNA populations are produced during spermatogenesis}

To characterize the piRNAs produced at these different stages of spermatogenesis, we performed deep sequencing of size-selected small RNAs ( 15-40 nt) from whole $y w$, bam, and bgcn testes, and from can and sa testes devoid of apical tips. We excluded the reads mapping to the rRNA, tRNAs, and snoRNAs. The reads in each library were normalized with three different methods: sequencing depth, relative log expression (RLE) method, and a normalization factor derived from Rfam RNAs (Burge et al. 2013; see details in Supplemental Information). Normalizations with all methods were comparable (Supplemental Table S1), and for the subsequent analyses in this study, we used RLE normalization unless otherwise noted. Like the small RNAs in ovaries, these small RNA libraries from testicular samples had a characteristic size peak ranging from 23 to $29 \mathrm{nt}$, indicating an enrichment of the piRNA population (Supplemental Table S1).

In order to characterize the piRNAs present in these libraries, we examined 23- to 29-nt reads mapping to canonical transposons, transposon insertions in the genome, and piRNA clusters. In general, we observed 23- to 29-nt reads mapping to canonical transposons and clusters were globally more abundant in spermatogonia libraries (bam and bgcn) compared with spermatocyte libraries (can and sa) (Fig. 2A). The spermatogonia libraries also had a higher number of piRNAs mapping to annotated transposons on the genome compared with those in primary spermatocytes: bam and bgcn libraries had an average of $27 \%$ of 23 - to 29 -nt sequences mapping to transposons compared with an average of $16.5 \%$ in can and sa libraries (Supplemental Fig. S4A). The higher amount of piRNAs in spermatogonia may have resulted from a general increase in piRNAs, or enrichment of piRNAs targeting certain transposons. The spermatogonia and primary spermatocytes represent different stages of spermatogenesis with differences in the spatial expression of PIWI family proteins (Fig. 1; Supplemental Figs. S1, S2). These results prompted us to examine for possible differences in piRNA populations between these samples. We used principal component analysis (PCA) to compare the piRNAs mapping to canonical transposons from testes enriched with different stages of germline cells (Fig. 2B). PCA grouped transposon-mapping piRNAs from spermatogonia samples, bam and bgcn, together, while those from primary spermatocyte samples, can and $s a$, were grouped together separately. This suggests that transposon-mapping-piRNA populations in individual mutants representing the same stage are alike and that transposon-mapping piRNAs from spermatogonia and primary spermatocyte samples are different. The transposon-mapping piRNAs from whole $y w$ testes showed greater similarity with primary spermatocytes samples (Fig. 2B), possibly because whole testes contain a large quantity of spermatocytes. Consistently with PCA, the Kolmogorov-Smirnov test (KS test) also confirmed the congruence between the transposon-mapping piRNA populations in the mutants representing the same stage (Supplemental Fig. S4B). These results suggest that male germline cells at different stages, spermatogonia and primary spermatocyte are enriched with piRNAs mapping to distinct transposon families.

Indeed, a detailed examination of piRNAs confirms differences in enrichment of piRNAs mapping to different transposon families between spermatogonia and primary spermatocyte samples.

Among the 99 canonical transposon families examined, anti-sense piRNAs that mapped to most of those were more enriched in spermatogonia (73/99) (Fig. 2C, red dots; Supplemental Table S2). Highly abundant piRNAs targeting transposon families such as INE-1, gypsy5, rover, Baril, and HMS-Beagle were more enriched in the spermatogonia samples (Supplemental Fig. S4E, upper panel), suggesting that the production of anti-sense piRNAs takes place more actively in spermatogonia. However, 14 transposon families including aurora-element, Dsim-ninja, opus, and baggins had more piRNAs mapping to those in primary spermatocytes than spermatogonia (14/99) (Fig. 2C, blue dots; Supplemental Fig. S4E, bottom panel). This suggests that piRNA biogenesis might be dynamically modulated to suppress the transposons in a stage-specific manner. We also did not observe a significant change in overall sense/antisense ratios for any transposon family between different samples (Fig. 2D; Supplemental Fig. S4C), suggesting that our observations were not influenced by accumulation of degradation products or sense piRNAs.

In addition to transposon-mapping piRNAs, spermatogonia libraries ( $b a m$ and $b g c n$ ) also had more cluster-mapping 

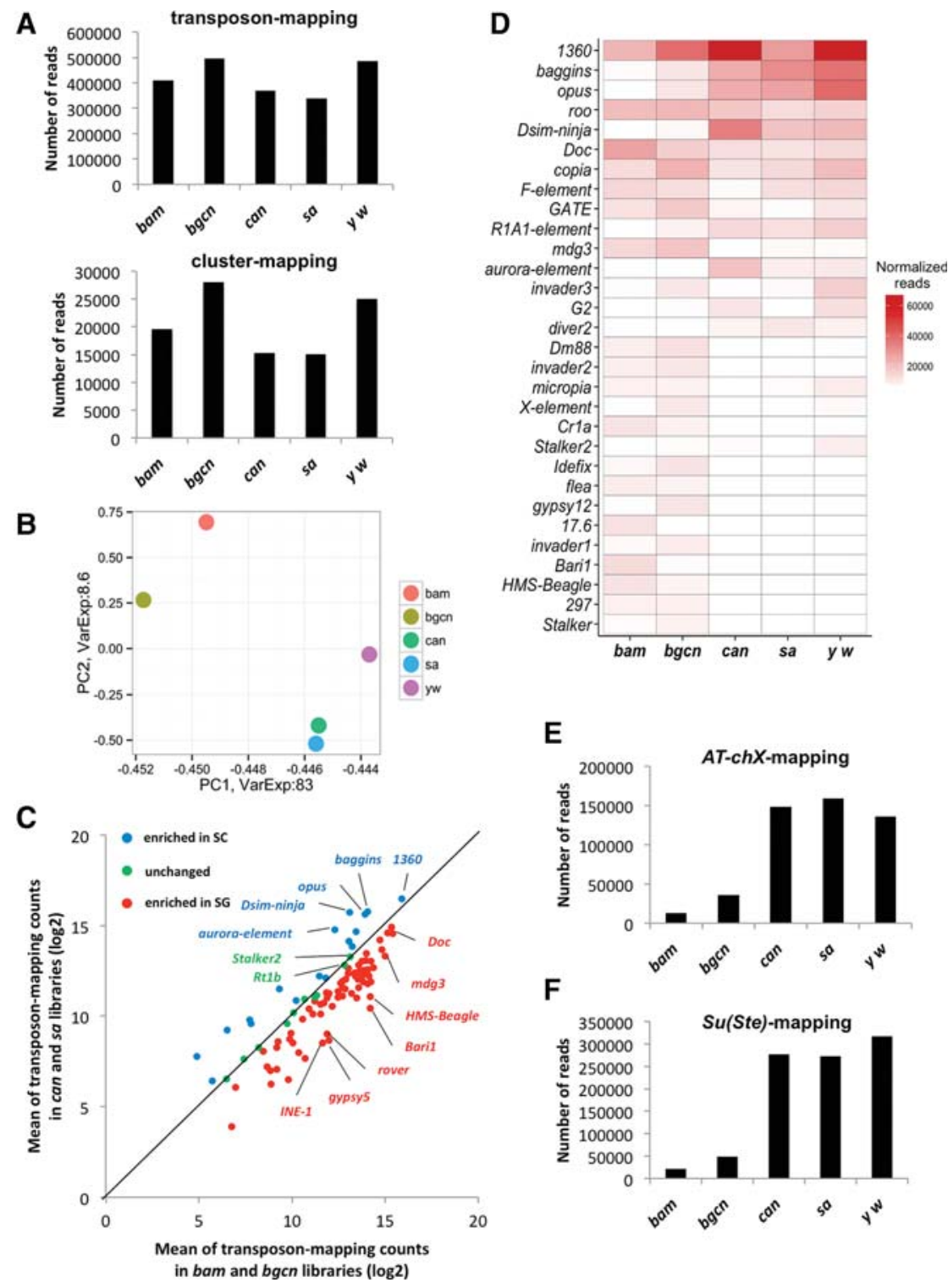

F

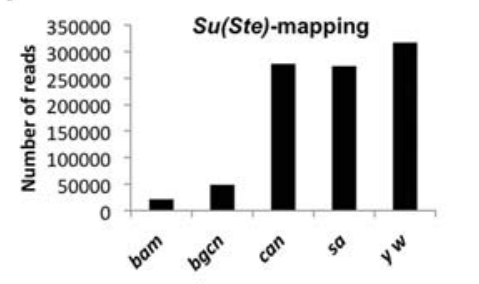

FIGURE 2. Distinct piRNA populations are produced during spermatogenesis. (A) The normalized numbers of 23- to 29-nt reads mapping to canonical transposons and clusters in bam, bgcn, $c a n, s a$, and $y w$ libraries. Transposon and cluster-mapping piRNAs were globally more abundant in bam and bgcn testes. (B) Principal component analysis of transposon-mapping piRNAs in each different type of arrested mutant testes. While piRNAs in can and sa mutants, and those in bam and bgcn exhibit similarity, respectively, they are quite different compared with the other group. $(C)$ Scatter-plot representing the expression of transposon-mapping piRNAs in spermatogonia versus primary spermatocytes. $\log _{2}$ of the mean number of reads of piRNAs mapping to each transposon families in bam and bgcn mutant testes are plotted on the $x$-axis, and those in can and sa mutant testes are plotted on the $y$-axis. piRNAs mapping to most of the transposon families are more abundant in spermatogonia (red dots) while some others are more abundant in primary spermatocytes (blue dots). (D) Heat-map representing the normalized expression levels of the most abundant transposon-mapping piRNAs (minimum in white, maximum in red) in bam, bgcn, can, sa, and $y w$ libraries. Most of the transposon-mapping piRNAs were enriched in bam and $b g c n$ libraries while some others show different trends. (E) The normalized number of AT-chX piRNAs in the indicated libraries. $(F)$ The normalized number of $S u(S t e)$ piRNAs in the indicated libraries. Both $S u(S t e)$ and $A T-c h X$ piRNAs were highly enriched in can and sa libraries.

piRNAs in comparison to those in the primary spermatocyte libraries (can and sa) (Fig. 2A). Notably, testicular cluster mapping-piRNAs exhibited distinct trends compared with those in ovarian piRNAs. In ovaries, piRNAs mapping to the $42 A B$ cluster contributes to $32 \%-46 \%$ among cluster-mapping piRNAs derived from the top 15 piRNA clusters (Supplemental Fig. S5A; data taken from Malone et al. 2009; Patil et al. 2014). In contrast, in developmentally arrested mutant and wild-type testes, $42 A B$-derived piRNAs contributed only to $6 \%-15 \%$ among the top 15 clusters (Supplemental Fig. S5A). Two bidirectional clusters at $38 \mathrm{C}$ contributed to the maximum amount piRNAs, between $28 \%$ and $42 \%$ among the top 15 clusters in testicular libraries, while these clusters contribute only $5 \%-7 \%$ among the 15 most active clusters in the ovarian libraries (Supplemental Figs. S5B, S6; Supplemental Table S3). These clusters, at $38 \mathrm{C}$, house transposons such as 1360, invader, GATE, and G2, and piRNAs mapping to those transposons were highly enriched in all testicular samples (Fig. 2C,D; Supplemental Table S3). The difference in the abundance of cluster-derived piRNAs between male and female gonads implicates a sex-dependent mechanism for piRNA production to combat with distinct populations of transposons in each gonad. Although testicular piRNAs mapping to a majority of clusters were more abundant in the spermatogonia libraries than those in the primary spermatocytes, piRNAs mapping to a few clusters, such as those at 3LHet and $\mathrm{Chr} \mathrm{U}$, were more enriched in the primary spermatocyte libraries, can and sa (Supplemental Fig. S5B). These results suggest that the generation of piRNAs from clusters may be temporally regulated during spermatogenesis.

It is known that two unique species of piRNAs, $\mathrm{Su}(\mathrm{Ste})$, and $A T$-chX piRNAs, are expressed abundantly in Drosophila testes (Nishida et al. 2007; Nagao et al. 2010). They are the most abundant piRNAs associated with Aub in Drosophila testes (Nagao et al. 2010). In stark contrast to the transposon- and clustermapping piRNAs, both $\mathrm{Su}(\mathrm{Ste})$ and $A T$ $\operatorname{ch} X$ piRNAs were highly enriched in the spermatocyte can and sa libraries (Fig. $2 \mathrm{E}, \mathrm{F})$, suggesting that the production of those distinct classes of piRNAs is highly regulated during the development of the male germline. In addition, the vast majority of $S u(S t e)$ and 
$A T-\operatorname{ch} X$ piRNAs were in antisense orientation (Supplemental Fig. S5C,D). This further supports that $\mathrm{Su}(\mathrm{Ste})$ and $A T$ chX piRNAs are produced by the primary piRNA pathway and that biogenesis of these piRNAs via the ping-pong cycle is very unlikely (Nagao et al. 2010). The unique expression patterns of Aub and Ago3 during spermatogenesis, and the distinct abundances of cluster-, transposon-, Su(Ste)-, and AT-chX-mapping piRNAs in spermatogonia and primary spermatocytes indicate that piRNA biogenesis is temporally regulated in a stage-specific manner.

\section{piRNAs with ping-pong signature are more abundant in spermatogonia}

In the female germline, secondary amplification involves both Aub and Ago3, and is fuelled by cleaved transposon transcripts to produce abundant piRNA quantities (Brennecke et al. 2007; Li et al. 2009). To examine the prevalence of the ping-pong cycle during spermatogenesis, we calculated ping-pong ratios of sense and antisense piRNAs pairs with 10 -nt overlaps to overall piRNAs (Fig. 3). The spermatogonia transposons-mapping piRNAs from bam and $b g c n$ testes contained more ping-pong piRNAs compared with the primary spermatocyte transposons-mapping piRNAs from can and sa testes (41\% and 40\% in bam and $b g c n$, respectively, versus $19 \%$ and $32 \%$ in can and $s a$, respectively; Fig. 3A). We further analyzed the ping-pong $z$-score for piRNAs mapping to 79 transposon families; small RNAs mapping to these constitute $>90 \%$ of transposon-mapping piRNAs. The ping-pong $z$-scores for piRNAs mapping to those transposon families were clearly higher globally in the spermatogonia samples than those in primary spermatocyte samples (Supplemental Fig. S7A). Consistently, piRNAs mapping to 29 transposon families in spermatogonia showed higher ping-pong $z$-scores than those in primary spermatocyte samples (Supplemental Fig. S7B). The higher ping-pong $z$ scores indicate more robust ping-pong amplification of piRNAs in spermatogonia stages. The can and sa mutant testes devoid of the apex express only Aub but not Ago3 contained piRNAs with a ping-pong signature (Figs. 1, 3). These results indicate that piRNA production via the ping-pong cycle takes place more actively in spermatogonia, and to a lesser extent in primary spermatocytes where Ago3 expression is not detectable.
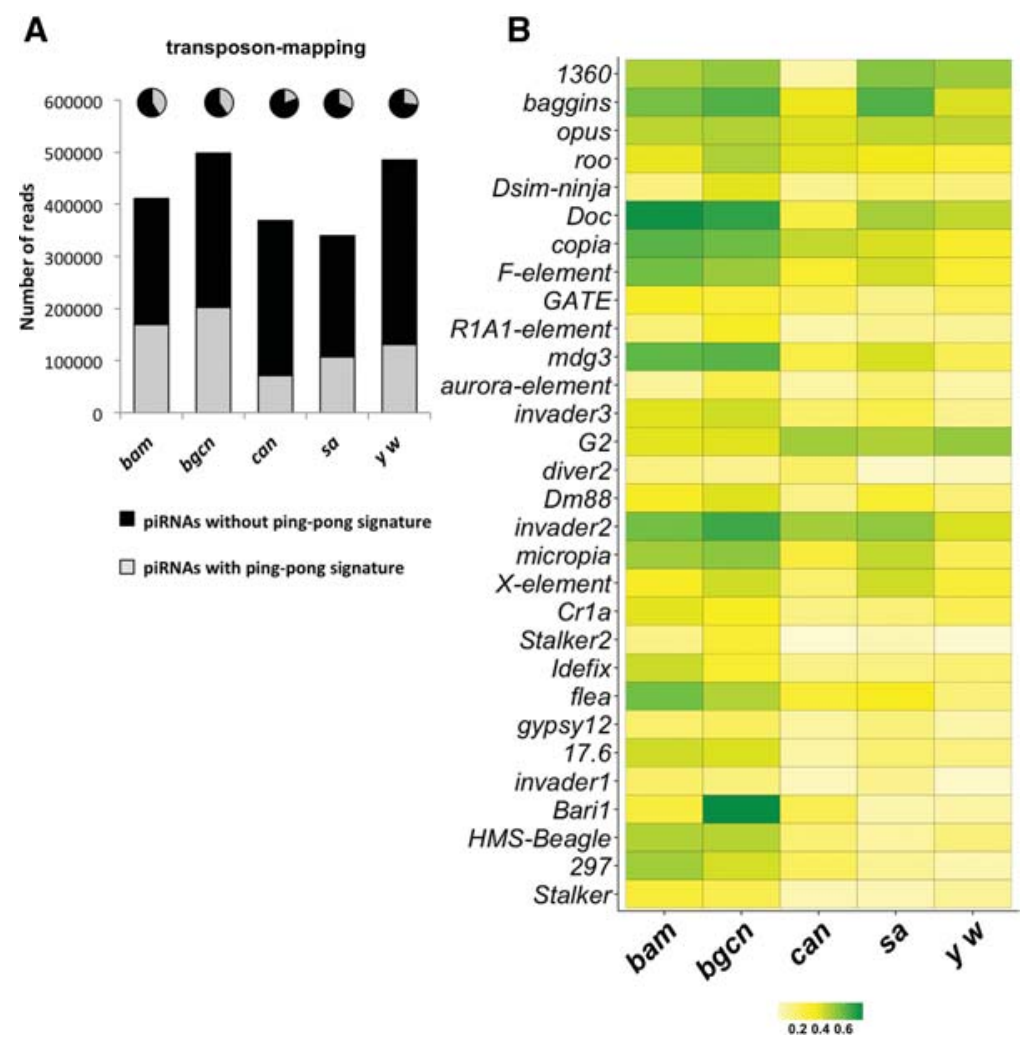

FIGURE 3. piRNAs with ping-pong signature are more abundant in spermatogonia but still detected in primary spermatocytes. (A) Bar graph showing the normalized numbers of transpoping-pong signature and with a ping-pong signature, respectively. The pie charts represent the percentage of those piRNAs in the total population of transposon-mapping piRNAs. piRNAs with ping-pong signature were more abundant in bam and $b g c n$ libraries but still detected in piRNAs (minimum in white, maximum in green) in the indicated libraries. For most transposon-mapping piRNA families, the ping-pong ratios are higher in spermatogonia (bam and bgcn libraries) compared with primary spermatocytes (can and sa libraries).

We speculated that the piRNAs harboring a ping-pong signature in primary spermatocytes could arise de novo from a noncanonical secondary amplification. Alternatively, they may have been generated in spermatogonia expressing both Aub and Ago3, and passed on while spermatogonia differentiate into primary spermatocytes. For the majority of transposon families, the ping-pong ratios were higher in spermatogonia (bam and bgcn libraries) compared with primary spermatocytes (can and sa libraries) (Fig. 3B; Supplemental Fig. S7B). However, there were some exceptions: For some specific transposon families such as roo, baggins, and opus, a higher ping-pong ratio was seen in primary spermatocytes compared with spermatogonia. In addition, the ping-pong $z$-scores for baggins and roo piRNAs were comparable between spermatogonia and primary spermatocyte samples (Supplemental Fig. S7B-F). These observations suggest that a noncanonical ping-pong cycle may act, reflected by an increase in ping-pong piRNAs for few transposons, in primary spermatocytes in an Ago3-independent manner. 


\section{A noncanonical ping-pong cycle likely takes place in Drosophila testes}

In order to examine whether a noncanonical ping-pong cycle takes place in the absence of Ago3 in the male germline, we deep-sequenced small RNAs from aub and ago3 mutants of loss-of-function alleles and their heterozygous testes (Fig. 4A; Supplemental Table S4;Li et al. 2009). In aub mutant testes, not only was Aub expression lost, but Ago3 expression

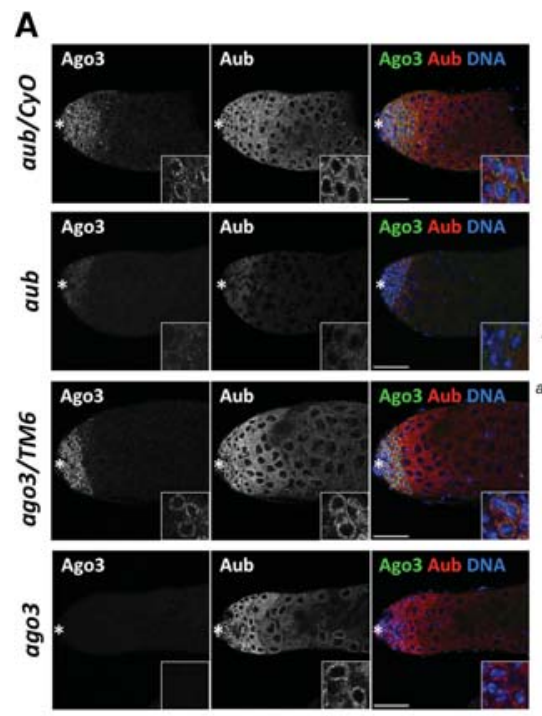

\section{C}

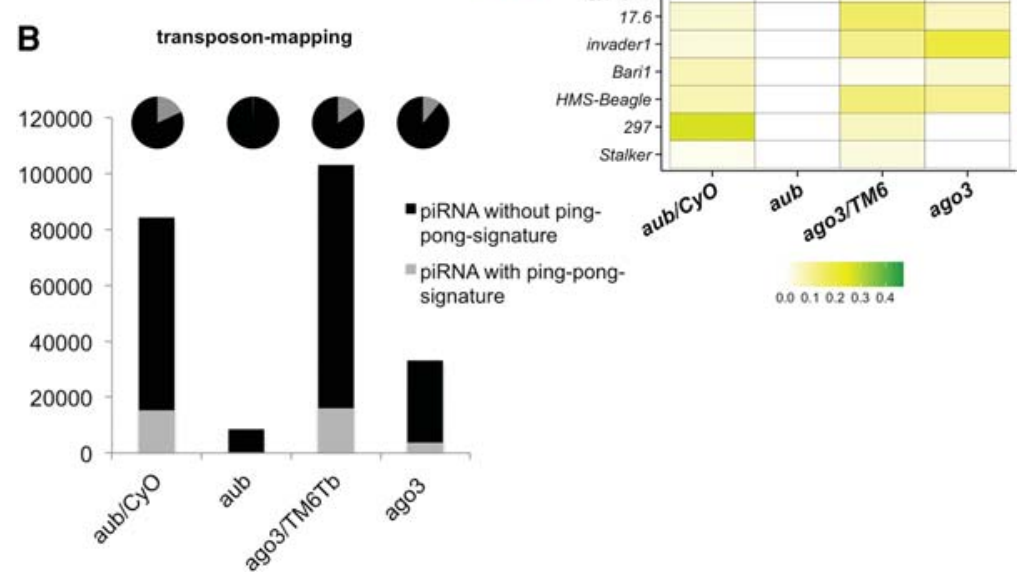

FIGURE 4. A noncanonical ping-pong amplification cycle takes place in Drosophila testes. $(A)$ Immunostaining of endogenous Aub and Ago3 in Drosophila testis of the indicated genotype. The asterisk denotes the anterior apex. Scale bars represent $50 \mu \mathrm{m}$. Insets show magnified cells from the GPC. In $a u b$ mutant testes, Aub and Ago3 expressions were significantly reduced. In ago3 mutant testes, Ago3 was lost but Aub was comparably expressed and localized to perinuclear nuage. $(B)$ Bar graph showing the normalized numbers of transposon-mapping piRNAs in the indicated libraries. Black and gray bars represent those without a ping-pong signature and with a ping-pong signature, respectively. The pie charts represent the percentage of those piRNAs in the total population of transposon-mapping piRNAs. Transposon-mapping piRNAs with a ping-pong signature were totally lost in aub mutant testes, while a small fraction of them was still present in ago3 mutants. (C) Heat-map representing the ping-pong ratios of the transposonmapping piRNAs (minimum in white, maximum in green) in the indicated libraries. In aub mutant testes, piRNAs containing ping-pong signatures are significantly reduced for almost all transposon families. Ago3 loss did not cause as severe a loss in ping-pong signatures for most transposon-mapping piRNAs as observed for that in aub mutants. was also significantly reduced. In contrast, in ago3 mutant testes, we did not observe a detectable change in Aub expression levels (Fig. 4A). In addition, even in the absence of Ago3, Aub was found to localize to perinuclear nuage (Fig. $4 \mathrm{~A})$. In $a u b$ mutant testes, where Ago 3 expression is also perturbed, the total number of transposon-mapping piRNAs was severely reduced (Supplemental Fig. S8A; 88\% reduction compared with heterozygous control). The reduction of those piRNAs in ago3 mutant testes was milder than that in aub mutants (Supplemental Fig. S8A; $77 \%$ reduction compared with heterozygous control). The aub heterozygous testes contained a lower sense/antisense piRNA ratio compared with that in ago3 heterozygous testes. This result may indicate that loss of a single aub copy could also mildly affect piRNA biogenesis. However, in $a u b$ mutant testes, transposon-mapping sense piRNAs were almost lost (96\% loss; Supplemental Table S4), while in ago3 mutant testes, both sense and antisense piRNAs were comparably affected. Hence the sense/antisense ratio of transposon-mapping piRNAs appears to be more severely affected in $a u b$ mutant testes (Supplemental Fig. S8A). We further examined piRNAs mapping to each transposon family in the mutant testes (Supplemental Fig. S8B). Evidently, piRNAs mapping to all transposon families are almost lost in $a u b$ mutant testes, while in ago3 mutants, piRNAs mapping to some transposon families still remained. Collectively, these results suggest that in ago3 mutant testes, Aub alone is likely to retain some activity to produce the piRNAs. Next, we analyzed the effect of aub and ago3 loss on $\mathrm{Su}$ (Ste) and $A T-\operatorname{chX}$ piRNAs. In $a u b \mathrm{mu}-$ tant testes where both Aub and Ago3 expression are perturbed, $S u(S t e)$ and $A T$ chX piRNAs are almost lost. Consistent with piRNAs mapping to transposons, however, ago3 mutant testes still contained a small number of $\mathrm{Su}(\mathrm{Ste})$ and AT-chX piRNAs (Supplemental Fig. S8C,D). Our data suggest that both Aub and Ago3 are required for the sufficient production of these piRNAs. These results correlate with the severity of aub and ago3 male sterility phenotypes: while aub mutant males are totally sterile, ago3 males are semi-fertile (Schmidt et al. 1999; Li et al. 2009). 
Next, we examined ping-pong cycle activity for the transposon-mapping piRNAs in aub and ago3 mutant testes. Transposon-mapping piRNAs with a ping-pong signature were almost completely lost in $a u b$ mutant testes due to severe reduction in sense piRNAs, while a small fraction of them was still present in ago3 mutants (Fig. 4B). We further analyzed the ratios of ping-pong piRNAs with overall piRNAs for every transposon family (Fig. 4C). Expectedly, in $a u b$ mutant testes, the ping-pong ratios for almost all transposons were severely reduced, indicating that $a u b$ loss in testes causes severe perturbation in ping-pong amplification. In ago $3 \mathrm{mu}-$ tants, both overall piRNAs and ping-pong piRNAs were globally reduced. Loss of Ago3 resulted in the reduction of pingpong ratios for many transposons. However, ping-pong ratios for several transposon families such as baggins and invader2 did not show any significant change (Fig. 4C). Consistently, although the ping-pong $z$-scores were reduced for several transposon families, for a few transposon families such as baggins and invader the $z$-scores were not reduced even in the absence of Ago3 (Supplemental Fig. S8E). The presence of ping-pong piRNA in ago3 mutants could also explain the occurrence of piRNAs with a ping-pong signature in the wild-type primary spermatocyte where Ago3 expression is not detected (Figs. 1, 3).

\section{DISCUSSION}

In this study, we investigated the dynamics of the piRNA components and piRNA populations during spermatogenesis using mutant testes enriched with distinct stages of male germline cells. Our results indicated that distinct piRNA populations are produced in spermatogonia and in primary spermatocytes. In general, higher amounts of transposonmapping piRNAs are present in spermatogonia, while $S u$ (Ste), AT-chX and a specific subset of transposon-mapping piRNAs are produced at higher levels in primary spermatocytes (Fig. 2; Supplemental Figs. S4,S5). These results suggest that piRNA production may be optimized in a stage-specific manner to potentially combat with different classes of transposons and repetitive sequences during male germline development. Consistent with the dynamics of Su(Ste) piRNAs expression, Ste protein is depressed and its crystals are accumulated in primary spermatocyte stages (Meyer et al. 1961). Previous studies showed that $\mathrm{Su}(\mathrm{Ste})$ piRNAs are derived from the Y chromosome, which is silenced as heterochromatin in most cell types except for primary spermatocytes (Pimpinelli et al. 1976; Aravin et al. 2004). Therefore, we speculate that the production of precursor transcripts of $S u$ (Ste) piRNAs from the Y chromosome takes place predominantly in primary spermatocytes. The production of $\mathrm{Su}$ (Ste) piRNAs derived from the $\mathrm{Y}$ chromosome might have evolved to be maximized in primary spermatocytes in order to combat with their target element, Ste. It is known that $\mathrm{Y}$ chromosome heterochromatin opens up for active transcription in primary spermatocytes (Bonaccorsi et al. 1988). Hence any other piRNAs originated from the Y-chromosome may be actively produced in primary spermatocytes. However, our attempts to map these piRNAs onto the Y chromosome failed due to the poor annotation of the $\mathrm{Y}$ chromosome sequence.

Our study also demonstrated that male piRNA production and processing mechanisms differ from that in the female germline. The most active cluster in the female germline, $42 A B$, is not very active during spermatogenesis; rather, many of the cluster-derived male piRNAs come from two clusters at 38C (Supplemental Figs. S5, S6). These results suggest that cluster piRNAs are produced in a sex-dependent manner. In addition, several distinct clusters produce more piRNAs in each different type of male germline cells, spermatogonia, and primary spermatocytes, further supporting a differential spatial and temporal dynamic of piRNA production during spermatogenesis (Supplemental Fig. S5). The clusters producing a high amount of piRNAs in testes enriched with spermatogonia house transposons against which an increased amount of ping-pong piRNAs were present in the spermatogonia samples, suggesting that cluster-derived piRNAs may trigger active piRNA production via ping-pong (Fig. 2; Supplemental Table S3). Molecular mechanism of stage- or sex-specific piRNA production remains an open question. Temporal regulation of piRNA production and distinct expression patterns of PIWI family proteins were also reported in mouse (Deng and Lin 2002; Kuramochi-Miyagawa et al. 2004, 2008; Aravin et al. 2007, 2008; Gan et al. 2011). In mouse spermatogonia, only MILI is expressed, while MILI and MIWI expression is observed in the pachytene spermatocytes and later stages (Deng and Lin 2002; KuramochiMiyagawa et al. 2004). In addition, two distinct piRNA populations are produced during mouse spermatogenesis, the pre-pachytene and pachytene piRNAs (Aravin et al. 2007, 2008; Kuramochi-Miyagawa et al. 2008; Gan et al. 2011). Similar to what we observed in Drosophila spermatogonia, pre-pachytene piRNAs contain more transposon-derived sequences compared with the pachytene piRNAs. Higher amounts of piRNAs in the early stages of male germline cells in both flies and mammals suggest that transposons are likely more poised toward active offense in the early stages of germline cells. In contrast, the piRNA-mediated response against some coding elements, such as Ste, is observed in later stages. These observations indicate that transposon activity and the protection mechanism might be evolutionarily conserved.

We further revealed that large amounts of ping-pong piRNAs are expressed in spermatogonia samples, but abundant $S u(S t e)$ and $A T$-chX piRNAs in primary spermatocytes are devoid of the ping-pong signature (Figs. 2, 3). This difference in the prevalence of ping-pong piRNAs during spermatogenesis also supports distinct piRNA biogenesis in each stage. Indeed, two PIWI family proteins required for the ping-pong cycle, Aub and Ago3, are expressed in spermatogonia, while only Aub but not Ago3 is detected in primary spermatocytes where fewer piRNAs with a ping-pong signature are seen (Fig. 1). However, the ping-pong cycle is yet 
noticeable in primary spermatocytes for a specific subset of transposon-mapping piRNAs (Fig. 3; Supplemental Fig. S7). This result suggests that a noncanonical ping-pong cycle acts in primary spermatocytes in an Ago3-independent manner. Indeed, ping-pong piRNAs remain present, albeit at lower levels, in ago3 mutant testes expressing Aub alone (Fig. 4; Supplemental Fig. S8), further supporting an Ago3-independent ping-pong cycle in the testicular germline. Taking clues from the female germline, Aub might mediate a homotypic ping-pong cycle in the absence of Ago 3 in primary spermatocytes (Zhang et al. 2011). However, there may be a possibility of new players in the male germline piRNA biogenesis. The next challenge will aim at dissecting the functions of other piRNA pathway components for production of piRNAs and their precursors in a stage-specific manner during spermatogenesis.

In contrast to the female germline where the PIWI family proteins are expressed throughout oogenesis, we confirmed that Aub and Ago3 exhibit distinct spatial expression during spermatogenesis. In spermatogonia, both are expressed, while in the primary spermatocyte, only Aub can be detected. Using developmentally arrested mutant testes, we observed a higher abundance of overall piRNA levels in spermatogonia than primary spermatocyte stages. However, piRNAs mapping to selective transposons and clusters were more abundant in the primary spermatocyte stages compared with spermatogonia stages. In addition, a significant amount of $\mathrm{Su}(\mathrm{Ste})$ and $A T$-chX mapping piRNAs is present in primary spermatocyte stages compared with that in spermatogonia, further indicating a temporal regulation of the piRNA pathway during Drosophila spermatogenesis. Although the primary spermatocytes lack Ago3 expression, they still retain piRNAs containing a ping-pong signature. Consistently, ago3 mutant testes also contain ping-pong piRNAs, supporting noncanonical Ago3-independent piRNA amplification in spermatogenesis. Our study shows that piRNA biogenesis is modulated during spermatogenesis to silence the different kinds of transposons and repetitive elements that are poised to be expressed during different stages of spermatogenesis.

\section{MATERIALS AND METHODS}

\section{Fly strains}

Drosophila melanogaster strains and alleles used were $y w$ (wild-type control unless otherwise stated), $a u b^{N 11}, a u b^{H N 2}$ (Schupbach and Wieschaus 1991; Wilson et al. 1996), ago ${ }^{t 2}$, ago $3^{t 3}$ (Li et al. 2009), $b_{a m}{ }^{\Delta 86}$ (McKearin and Spradling 1990), bgcn ${ }^{Q S 2}$ (Schupbach and Wieschaus 1991), $\operatorname{can}^{12}$ (Hiller et al. 2001), and $s a^{2}$ (Lin et al. 1996). They were grown on standard cornmeal-agar medium at $25^{\circ} \mathrm{C}$.

\section{Immunostaining}

Antibodies that were used for immunostaining were anti-Ago3 (mouse, 1:200) (Lim et al. 2009), anti-Aub (rabbit, 1:300; a kind gift from Dr. Paul Lasko) (Lim and Kai 2007), anti-Hts (mouse, 1:20; DSHB), anti-S5 (mouse, 1:5; a kind gift from Dr. Harald Saumweber) (Saumweber et al. 1980), anti-Ste (rabbit, 1:1000; a kind gift from Dr. William Theurkauf) (Klattenhoff et al. 2007), anti-Lam (mouse, 1:5; DSHB). Alexa Fluor- (488, 555 or 633) conjugated goat anti-mouse and anti-rabbit immunoglobulin (1:200; Invitrogen) were used as secondary antibodies. Images were captured with a Zeiss LSM 510 META inverted confocal microscope and processed with Adobe Photoshop.

\section{Western blotting}

Testicular lysates were prepared as described previously (Drummond-Barbosa and Spradling 2004). The following primary antibodies were used: anti-Aub (mouse, 1:1000) (Nishida et al. 2007), anti-Ago3 (mouse, 1:500) (Gunawardane et al. 2007) and anti- $\alpha$-Tubulin (DM1A, mouse, 1:1000, Upstate). HRP-conjugated anti-mouse immunoglobulin (1:5000; Bio-Rad) was used as the secondary antibody.

\section{Small RNA sequencing and analysis}

RNA isolation, small RNA library preparation, and deep-sequencing analysis from testes are described in detail in the Supplemental Information. The libraries are deposited in the GEO database and can be accessed with accession number GEO: GSE69417.

\section{SUPPLEMENTAL MATERIAL}

Supplemental material is available for this article.

\section{ACKNOWLEDGMENTS}

We are grateful to Dr. Paul Lasko, Dr. Harald Saumweber, and Dr. William Theurkauf for providing Aub, S5, and Ste antibodies, respectively. We are also grateful to Dr. Margaret T. Fuller and Bloomington Drosophila Stock Center for kindly providing fly stocks. We thank Kai laboratory members and Dr. Joanne Yew for critical reading, discussions, and suggestions. This work was supported by Temasek Life Sciences Laboratory and Singapore Millennium Foundation, and partly by JSPS KAKENHI grant number 26291048 to T.K.

Received January 18, 2016; accepted April 15, 2016.

\section{REFERENCES}

Anand A, Kai T. 2012. The tudor domain protein kumo is required to assemble the nuage and to generate germline piRNAs in Drosophila. EMBO J 31: 870-882.

Aravin AA, Naumova NM, Tulin AV, Vagin VV, Rozovsky YM, Gvozdev VA. 2001. Double-stranded RNA-mediated silencing of genomic tandem repeats and transposable elements in the D. melanogaster germline. Curr Biol 11: 1017-1027.

Aravin AA, Klenov MS, Vagin VV, Bantignies F, Cavalli G, Gvozdev VA. 2004. Dissection of a natural RNA silencing process in the Drosophila melanogaster germ line. Mol Cell Biol 24: 6742-6750.

Aravin A, Gaidatzis D, Pfeffer S, Lagos-Quintana M, Landgraf P, Iovino $\mathrm{N}$, Morris $\mathrm{P}$, Brownstein MJ, Kuramochi-Miyagawa S, 
Nakano T, et al. 2006. A novel class of small RNAs bind to MILI protein in mouse testes. Nature 442: 203-207.

Aravin AA, Sachidanandam R, Girard A, Fejes-Toth K, Hannon GJ. 2007. Developmentally regulated piRNA clusters implicate MILI in transposon control. Science 316: 744-747.

Aravin AA, Sachidanandam R, Bourc'his D, Schaefer C, Pezic D, Toth KF, Bestor T, Hannon GJ. 2008. A piRNA pathway primed by individual transposons is linked to de novo DNA methylation in mice. Mol Cell 31: 785-799.

Bonaccorsi S, Pisano C, Puoti F, Gatti M. 1988. Y chromosome loops in Drosophila melanogaster. Genetics 120: 1015-1034.

Bozzetti MP, Massari S, Finelli P, Meggio F, Pinna LA, Boldyreff B, Issinger OG, Palumbo G, Ciriaco C, Bonaccorsi S, et al. 1995. The Ste locus, a component of the parasitic cry-Ste system of Drosophila melanogaster, encodes a protein that forms crystals in primary spermatocytes and mimics properties of the $\beta$ subunit of casein kinase 2. Proc Natl Acad Sci 92: 6067-6071.

Brennecke J, Aravin AA, Stark A, Dus M, Kellis M, Sachidanandam R, Hannon GJ. 2007. Discrete small RNA-generating loci as master regulators of transposon activity in Drosophila. Cell 128: 1089-1103.

Burge SW, Daub J, Eberhardt R, Tate J, Barquist L, Nawrocki EP, Eddy SR, Gardner PP, Bateman A. 2013. Rfam 11.0: 10 years of RNA families. Nucleic Acids Res 41: D226-D232.

Chen X, Hiller M, Sancak Y, Fuller MT. 2005. Tissue-specific TAFs counteract Polycomb to turn on terminal differentiation. Science 310: 869-872.

Cox DN, Chao A, Lin H. 2000. piwi encodes a nucleoplasmic factor whose activity modulates the number and division rate of germline stem cells. Development 127: 503-514.

Deng W, Lin H. 2002. miwi, a murine homolog of piwi, encodes a cytoplasmic protein essential for spermatogenesis. Dev Cell 2: 819-830.

Drummond-Barbosa D, Spradling AC. 2004. a-Endosulfine, a potential regulator of insulin secretion, is required for adult tissue growth control in Drosophila. Dev Biol 266: 310-321.

Eddy EM. 1975. Germ plasm and the differentiation of the germ cell line. Int Rev Cytol 43: 229-280.

Findley SD, Tamanaha M, Clegg NJ, Ruohola-Baker H. 2003. Maelstrom, a Drosophila spindle-class gene, encodes a protein that colocalizes with Vasa and RDE1/AGO1 homolog, Aubergine, in nuage. Development 130: 859-871.

Fuller MT. 1993. Spermatogenesis. In Development of Drosophila melanogaster (ed. Martinez-Arias M, M Bate), pp. 71-147. Cold Spring Harbor Press, Cold Spring Harbor, NY.

Gan H, Lin X, Zhang Z, Zhang W, Liao S, Wang L, Han C. 2011. piRNA profiling during specific stages of mouse spermatogenesis. RNA 17: 1191-1203.

Girard A, Sachidanandam R, Hannon GJ, Carmell MA. 2006. A germline-specific class of small RNAs binds mammalian Piwi proteins. Nature 442: 199-202.

Gonczy P, Matunis E, DiNardo S. 1997. Bag-of-marbles and benign gonial cell neoplasm act in the germline to restrict proliferation during Drosophila spermatogenesis. Development 124: 43614371.

Grivna ST, Pyhtila B, Lin H. 2006. MIWI associates with translational machinery and PIWI-interacting RNAs (piRNAs) in regulating spermatogenesis. Proc Natl Acad Sci 103: 13415-13420.

Gunawardane LS, Saito K, Nishida KM, Miyoshi K, Kawamura Y, Nagami T, Siomi H, Siomi MC. 2007. A slicer-mediated mechanism for repeat-associated siRNA $5^{\prime}$ end formation in Drosophila. Science 315: $1587-1590$.

Hardy RW, Tokuyasu KT, Lindsley DL, Garavito M. 1979. The germinal proliferation center in the testis of Drosophila melanogaster. J Ultrastruct Res 69: 180-190.

Harris AN, Macdonald PM. 2001. Aubergine encodes a Drosophila polar granule component required for pole cell formation and related to eIF2C. Development 128: 2823-2832.
Hiller MA, Lin TY, Wood C, Fuller MT. 2001. Developmental regulation of transcription by a tissue-specific TAF homolog. Genes Dev 15: $1021-1030$.

Hiller M, Chen X, Pringle MJ, Suchorolski M, Sancak Y, Viswanathan S, Bolival B, Lin TY, Marino S, Fuller MT. 2004. Testis-specific TAF homologs collaborate to control a tissue-specific transcription program. Development 131: 5297-5308.

Kawaoka S, Izumi N, Katsuma S, Tomari Y. 2011. 3' end formation of PIWI-interacting RNAs in vitro. Mol Cell 43: 1015-1022.

Klattenhoff C, Bratu DP, McGinnis-Schultz N, Koppetsch BS, Cook HA, Theurkauf WE. 2007. Drosophila rasiRNA pathway mutations disrupt embryonic axis specification through activation of an ATR/ Chk2 DNA damage response. Dev Cell 12: 45-55.

Kotelnikov RN, Klenov MS, Rozovsky YM, Olenina LV, Kibanov MV, Gvozdev VA. 2009. Peculiarities of piRNA-mediated post-transcriptional silencing of Stellate repeats in testes of Drosophila melanogaster. Nucleic Acids Res 37: 3254-3263.

Kuramochi-Miyagawa S, Kimura T, Ijiri TW, Isobe T, Asada N, Fujita Y, Ikawa M, Iwai N, Okabe M, Deng W, et al. 2004. Mili, a mammalian member of piwi family gene, is essential for spermatogenesis. Development 131: 839-849.

Kuramochi-Miyagawa S, Watanabe T, Gotoh K, Totoki Y, Toyoda A, Ikawa M, Asada N, Kojima K, Yamaguchi Y, Ijiri TW, et al. 2008. DNA methylation of retrotransposon genes is regulated by Piwi family members MILI and MIWI2 in murine fetal testes. Genes Dev 22: 908-917.

Lau NC, Seto AG, Kim J, Kuramochi-Miyagawa S, Nakano T, Bartel DP, Kingston RE. 2006. Characterization of the piRNA complex from rat testes. Science 313: 363-367.

Le Thomas A, Rogers AK, Webster A, Marinov GK, Liao SE, Perkins EM, Hur JK, Aravin AA, Tóth KF. 2013. Piwi induces piRNA-guided transcriptional silencing and establishment of a repressive chromatin state. Genes Dev 27: 390-399.

Li C, Vagin VV, Lee S, Xu J, Ma S, Xi H, Seitz H, Horwich MD, Syrzycka M, Honda BM, et al. 2009. Collapse of germline piRNAs in the absence of Argonaute 3 reveals somatic piRNAs in flies. Cell 137: 509-521.

Liang L, Diehl-Jones W, Lasko P. 1994. Localization of vasa protein to the Drosophila pole plasm is independent of its RNA-binding and helicase activities. Development 120: 1201-1211.

Lim AK, Kai T. 2007. Unique germ-line organelle, nuage, functions to repress selfish genetic elements in Drosophila melanogaster. Proc Natl Acad Sci 104: 6714-6719.

Lim AK, Tao L, Kai T. 2009. piRNAs mediate posttranscriptional retroelement silencing and localization to pi-bodies in the Drosophila germline. J Cell Biol 186: 333-342.

Lin H, Yue L, Spradling AC. 1994. The Drosophila fusome, a germlinespecific organelle, contains membrane skeletal proteins and functions in cyst formation. Development 120: 947-956.

Lin TY, Viswanathan S, Wood C, Wilson PG, Wolf N, Fuller MT. 1996. Coordinate developmental control of the meiotic cell cycle and spermatid differentiation in Drosophila males. Development 122: $1331-1341$.

Malone CD, Brennecke J, Dus M, Stark A, McCombie WR, Sachidanandam R, Hannon GJ. 2009. Specialized piRNA pathways act in germline and somatic tissues of the Drosophila ovary. Cell 137: 522-535.

McClintock B. 1951. Chromosome organization and genic expression. Cold Spring Harb Symp Quant Biol 16: 13-47.

McKearin D, Ohlstein B. 1995. A role for the Drosophila bag-of-marbles protein in the differentiation of cystoblasts from germline stem cells. Development 121: 2937-2947.

McKearin DM, Spradling AC. 1990. bag-of-marbles: a Drosophila gene required to initiate both male and female gametogenesis. Genes Dev 4: 2242-2251.

Meyer GF, Hess O, Beermann W. 1961. [Phase specific function structure in spermatocyte nuclei of Drosophila melanogaster and their dependence of Y chromosomes] (article in German). Chromosoma 12: 676-716. 
Nagao A, Mituyama T, Huang H, Chen D, Siomi MC, Siomi H. 2010. Biogenesis pathways of piRNAs loaded onto AGO3 in the Drosophila testis. RNA 16: 2503-2515.

Nishida KM, Saito K, Mori T, Kawamura Y, Nagami-Okada T, Inagaki S, Siomi H, Siomi MC. 2007. Gene silencing mechanisms mediated by Aubergine piRNA complexes in Drosophila male gonad. RNA 13: 1911-1922.

Patil VS, Kai T. 2010. Repression of retroelements in Drosophila germline via piRNA pathway by the Tudor domain protein Tejas. Curr Biol 20: 724-730.

Patil VS, Anand A, Chakrabarti A, Kai T. 2014. The Tudor domain protein Tapas, a homolog of the vertebrate Tdrd7, functions in the piRNA pathway to regulate retrotransposons in germline of Drosophila melanogaster. BMC Biol 12: 61.

Pimpinelli S, Santini G, Gatti M. 1976. Characterization of Drosophila heterochromatin. II. C- and N-banding. Chromosoma 57: 377-386.

Risau W, Symmons P, Saumweber H, Frasch M. 1983. Nonpackaging and packaging proteins of hnRNA in Drosophila melanogaster. Cell 33: 529-541.

Sato K, Iwasaki YW, Shibuya A, Carninci P, Tsuchizawa Y, Ishizu H, Siomi MC, Siomi H. 2015. Krimper enforces an antisense bias on piRNA pools by binding AGO3 in the Drosophila germline. Mol Cell 59: 553-563.
Saumweber H, Symmons P, Kabisch R, Will H, Bonhoeffer F. 1980. Monoclonal antibodies against chromosomal proteins of Drosophila melanogaster: establishment of antibody producing cell lines and partial characterization of corresponding antigens. Chromosoma 80: 253-275.

Schmidt A, Palumbo G, Bozzetti MP, Tritto P, Pimpinelli S, Schafer U. 1999. Genetic and molecular characterization of sting, a gene involved in crystal formation and meiotic drive in the male germ line of Drosophila melanogaster. Genetics 151: 749-760.

Schupbach T, Wieschaus E. 1991. Female sterile mutations on the second chromosome of Drosophila melanogaster. II. Mutations blocking oogenesis or altering egg morphology. Genetics 129: 1119-1136.

Spradling AC, Nystul T, Lighthouse D, Morris L, Fox D, Cox R, Tootle T, Frederick R, Skora A. 2008. Stem cells and their niches: integrated units that maintain Drosophila tissues. Cold Spring Harb Symp Quant Biol 73: 49-57.

Wilson JE, Connell JE, Macdonald PM. 1996. Aubergine enhances oskar translation in the Drosophila ovary. Development 122: 1631-1639.

Zhang Z, Xu J, Koppetsch BS, Wang J, Tipping C, Ma S, Weng Z, Theurkauf WE, Zamore PD. 2011. Heterotypic piRNA Ping-Pong requires qin, a protein with both E3 ligase and Tudor domains. Mol Cell 44: 572-584. 

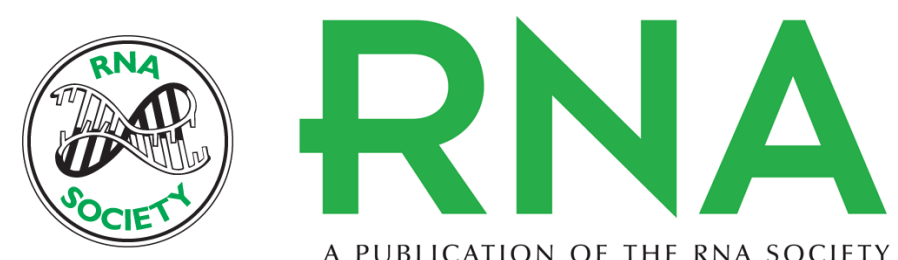

A PUBLICATION OF THE RNA SOCIETY

\section{The piRNA pathway is developmentally regulated during spermatogenesis in Drosophila}

Emilie Quénerch'du, Amit Anand and Toshie Kai

RNA 2016 22: 1044-1054 originally published online May 20, 2016

Access the most recent version at doi:10.1261/rna.055996.116

\section{Supplemental http://rnajournal.cshlp.org/content/suppl/2016/05/20/rna.055996.116.DC1 Material}

References This article cites 56 articles, 33 of which can be accessed free at: http://rnajournal.cshlp.org/content/22/7/1044.full.html\#ref-list-1

Creative This article is distributed exclusively by the RNA Society for the first 12 months after the Commons License full-issue publication date (see http://rnajournal.cshlp.org/site/misc/terms.xhtml). After 12 months, it is available under a Creative Commons License (Attribution-NonCommercial 4.0 International), as described at http://creativecommons.org/licenses/by-nc/4.0/.

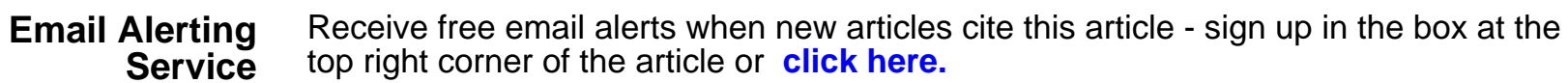

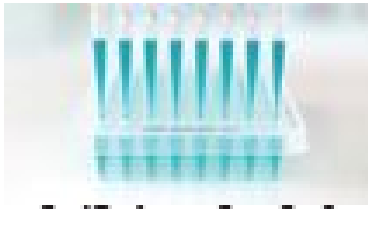

Providing Precise Solutions for your research.

To subscribe to RNA go to:

http://rnajournal.cshlp.org/subscriptions

(C) 2016 Quénerch'du et al.; Published by Cold Spring Harbor Laboratory Press for the RNA Society 